\title{
In vivo imaging of the nucleus of the solitary tract with Magnetization Transfer at 7 Tesla
}

Citation for published version (APA):

Priovoulos, N., Poser, B. A., Ivanov, D., Verhey, F. R. J., \& Jacobs, H. I. L. (2019). In vivo imaging of the nucleus of the solitary tract with Magnetization Transfer at 7 Tesla. Neuroimage, 201, [116071]. https://doi.org/10.1016/j.neuroimage.2019.116071

Document status and date:

Published: 01/01/2019

DOI:

10.1016/j.neuroimage.2019.116071

Document Version:

Publisher's PDF, also known as Version of record

Document license:

Taverne

Please check the document version of this publication:

- A submitted manuscript is the version of the article upon submission and before peer-review. There can be important differences between the submitted version and the official published version of record.

People interested in the research are advised to contact the author for the final version of the publication, or visit the DOI to the publisher's website.

- The final author version and the galley proof are versions of the publication after peer review.

- The final published version features the final layout of the paper including the volume, issue and page numbers.

Link to publication

\footnotetext{
General rights rights.

- You may freely distribute the URL identifying the publication in the public portal. please follow below link for the End User Agreement:

www.umlib.nl/taverne-license

Take down policy

If you believe that this document breaches copyright please contact us at:

repository@maastrichtuniversity.nl

providing details and we will investigate your claim.
}

Copyright and moral rights for the publications made accessible in the public portal are retained by the authors and/or other copyright owners and it is a condition of accessing publications that users recognise and abide by the legal requirements associated with these

- Users may download and print one copy of any publication from the public portal for the purpose of private study or research.

- You may not further distribute the material or use it for any profit-making activity or commercial gain

If the publication is distributed under the terms of Article $25 \mathrm{fa}$ of the Dutch Copyright Act, indicated by the "Taverne" license above, 


\title{
In vivo imaging of the nucleus of the solitary tract with Magnetization Transfer at 7 Tesla
}

\author{
Nikos Priovoulos $^{\text {a, }}$, Benedikt A. Poser ${ }^{\mathrm{b}}$, Dimo Ivanov ${ }^{\mathrm{b}}$, Frans R.J. Verhey ${ }^{\mathrm{a}}$, Heidi I.L. Jacobs ${ }^{\mathrm{a}, \mathrm{b}, \mathrm{c}}$ \\ ${ }^{a}$ Alzheimer Center Limburg, School for Mental Health and Neuroscience, Department of Psychiatry and Neuropsychology, Maastricht University, Maastricht, the \\ Netherlands \\ ${ }^{\mathrm{b}}$ Department of Cognitive Neuroscience, Faculty of Psychology and Neuroscience, Maastricht University, Maastricht, the Netherlands \\ ${ }^{\mathrm{c}}$ Division of Nuclear Medicine and Molecular Imaging, Department of Radiology, Massachusetts General Hospital/Harvard Medical School, Boston, MA, USA
}

\section{A R T I C L E I N F O}

\section{Keywords:}

Nucleus of the solitary tract

Magnetization transfer

Neuromelanin

Brainstem

Norepinephrine

$7 \mathrm{~T}$

MRI

\begin{abstract}
A B S T R A C T
The nucleus of the solitary tract (NTS) is a nuclei complex with, among others, a high concentration of noradrenergic neurons (including the noradrenergic subnuclei named A1 and A2) in the medulla. The NTS regulates several cognitive, neuroendocrine and autonomic functions. No method currently exists to anatomically visualize the NTS in vivo. Several noradrenergic and dopaminergic nuclei have been successfully imaged using Magnetization Transfer (MT) contrast manipulation. We therefore hypothesized that an efficient, high-resolution MTweighted sequence at $7 \mathrm{~T}$ might successfully image the NTS. In this study, we found a hyperintensity, similar to hyperintensities found in other noradrenergic and dopaminergic nuclei, consistent with the expected NTS location, and specific to the MT-weighted images. The localization of the hyperintensity was found to be consistent between individuals and slices and in good correspondence to a histological atlas and a meta-analytic map of fMRI-based NTS activation. We conclude that the method may, for the first time, achieve NTS imaging in vivo and within a clinically-feasible acquisition time. To facilitate NTS research at lower field strengths, an NTS template was created and made publicly available.
\end{abstract}

\section{Introduction}

The nucleus of the solitary tract (NTS) consists of a complex of grey matter nuclei in the medulla oblongata that regulate several functions, including respiration (Beckstead et al., 1980), heart rate (Schwaber et al., 1980) as well as gustatory (Jones et al., 2012; Little et al., 2014) and memory (Mello-Carpes and Izquierdo, 2013) functions. In recent years, the interest in in vivo examination of its function has been mounting (Frangos et al., 2015; Frangos and Komisaruk, 2017; Garcia et al., 2017; Jones et al., 2012; Little et al., 2014; Yakunina et al., 2016) but, to the best of our knowledge, there is no method to identify the nucleus anatomically.

Currently, dopaminergic and noradrenergic nuclei, such as the Substantia Nigra pars compacta (SNpc) and Locus Coeruleus (LC), are usually imaged at $3 \mathrm{~T}(\mathrm{~T})$ using a Turbo Spin Echo (TSE) sequence with a short echo time (TE), where these nuclei show a hyperintense signal (Langley et al., 2016; Sasaki et al., 2006). This hyperintensity is potentially related to the presence of neuromelanin occurring in these sites as a by-product of dopamine and noradrenaline synthesis (Keren et al., 2015). It has been suggested that this contrast is due to implicit Magnetization Transfer (MT) contrast and indeed MT-based approaches have been used to successfully image the noradrenergic LC (Nakane et al., 2008; Priovoulos et al., 2018). MT refers to the magnetization exchange between the macromolecular and free water pools. This exchange (and a subsequent reduction of signal due to saturation of the free water pool) is implicit in most MRI experiments, and particularly so in specific absorption rate (SAR) intensive acquisitions with a high level of RF power deposition such as TSE. Since the NTS complex shows high concentration of noradrenergic neurons (typically identified as the A1 and A2 noradrenergic subnuclei) (Naidich et al., 2009), we hypothesized that the NTS might be sensitive to MT contrast, similarly to the noradrenergic LC.

The human NTS is a small complex (approximately $1 \times 1 \times 10 \mathrm{~mm}^{3}$ ) (Naidich et al., 2009). Any attempts for structural imaging of the nucleus therefore requires sufficiently high resolution and a reasonably short acquisition time (TA) to reduce motion. Signal-to-noise ratio (SNR) gains at higher magnetic field $\left(\mathrm{B}_{0}\right)$ can facilitate these prerequisites. While

\footnotetext{
* Corresponding author. Alzheimer Center Limburg, School for Mental Health and Neuroscience, Department of Psychiatry and Neuropsychology, Maastricht University, Dokter Tanslaan 12, Room K.3.G3.057, 6229 ET, Maastricht, the Netherlands.

E-mail address: nikos.priovoulos@maastrichtuniversity.nl (N. Priovoulos).
} 
TSE/MT approaches are routinely used at 3T, high-resolution applications at UHF are challenging due to the high number of radio frequency (RF) pulses. The RF power increases approximately quadratically with $\mathrm{B}_{0}$, which results in increased SAR and hence need for prolongation of acquisition time (TA); which in turn reduce the impact of SNR gained per unit time. Recently, we applied an efficient MT-Turbo Flash (MT-TFL) (Mougin et al., 2010, 2013) application to image the noradrenergic LC at high-resolution and in a clinically tolerable TA (Priovoulos et al., 2018). This made use of a fast turbo flash gradient echo readout (versus spin echo in TSE), following an intensive off-resonance saturation (instead of a single MT pulse interleaved with a gradient echo readout) to allow high resolution MT-weighted images at UHF. In this study, we hypothesized that an efficient MT-weighted scheme with high resolution and a fast TA, such as MT-TFL, would allow visualization of the NTS as a hyperintensity. We examined this hypothesis by acquiring in vivo MT-TFL scans of the brainstem and comparing the specificity of the contrast mechanism and localization to functional and atlas markers of NTS.

\section{Methods}

MR scans were performed on a 7T Magnetom Siemens scanner (Siemens Healthineers, Erlangen, Germany) with a 32-channel head coil (Nova Medical, Wilmington, MA, USA).

\subsection{Pilot experiment}

Five participants (three females; 21-24 years old) were recruited for a pilot experiment, to determine if the NTS showed sensitivity to MT manipulation and whether this was specific to the MT. All participants provided written informed consent. Approval of the experimental protocol was obtained from the local ethical committee of the Faculty of Psychology and Neuroscience at Maastricht University. All participants were right-handed. An in-house developed MT-TFL sequence was performed to attempt to image the NTS at high resolution. The sequence consisted of a multi-shot 3D readout $(\mathrm{TR}=538 \mathrm{~ms}$, TE $=4.08 \mathrm{~ms}$, flip angle $=8^{\circ}$, voxel size $=0.4 \times 0.4 \times 0.5 \mathrm{~mm}^{3}, \quad F O V=192 \times 192 \times 60$, partial Fourier $=6 / 8$, no parallel imaging factor) with center-out k-space sampling, preceded by 20 off-resonant Gaussian pulses (pulse length $=5.12 \mathrm{~ms}$, bandwidth $=250 \mathrm{~Hz}$, individual pulse $\mathrm{B}_{1}=3.322 \mu \mathrm{T}$ $\left(260.88^{\circ}\right)$.

Continuous wave power equivalent (CWPE) over the train duration $=0.084 \mu \mathrm{T}\left(193.23^{\circ}\right)$ (Ramani et al., 2002), offset $\left.=2000 \mathrm{~Hz}\right)$. These settings have been found to be optimal in imaging the LC and SNpc; for the sequence schema, see S-Fig. 3 in (Priovoulos et al., 2018). The field-of-view (FOV) was oriented approximately perpendicular to the pons and placed so that the obex (caudal border of the 4th ventricle) coincided with the inferior part of the FOV. This was chosen, since the caudal part of NTS is superior to the obex and thus ensured NTS inclusion (Naidich et al., 2009). A matched TFL sequence, but with the MT pulses turned off, was similarly acquired to check the specificity to MT contrast; an MTR map $\left(M T R=\frac{M T T F L}{T F L}\right)$ was extracted. Finally, a Magnetization Prepared 2 Rapid Acquisition Gradient Echoes (MP2RAGE) sequence (Marques et al., 2010) for whole brain imaging (TR $=5000 \mathrm{~s}$, $\mathrm{TE}=2.47 \mathrm{~ms}$, flip angle $=5^{\circ} / 3^{\circ}$, voxel size $=0.7 \mathrm{~mm}^{3}$, number of slices $=240$ ) was obtained. A Proton Density (PD) like image and $\mathrm{T}_{1}$ map were extracted from the MP2RAGE and resliced to the NTS space. The MT-TFL, TFL, PD and $\mathrm{T}_{1}$ map were compared at the level of medulla for possible contrast consistent with NTS placement; a hyperintensity consistent with NTS was found in the MT-TFL scan.

\subsection{Main dataset}

To reproduce our results and confirm the localization of the finding, we acquired an additional dataset within the context of another study. This dataset consisted of an MT-TFL and an MP2RAGE, as described above, acquired in 19 healthy young individuals. Four participants were excluded due to motion corruption, leaving 15 individuals (7 female; 21-26 years old). This dataset was merged with the pilot data for a grouplevel analysis (total $\mathrm{N}=20$ ).

\subsubsection{Template creation}

Registration of the MT-TFL images to a common template was not trivial: the limited FOV and the curvature of the lower brainstem resulted in heterogeneity of anatomical landmarks outside the brainstem, which could guide the registration. At the same time, estimation of robust warp fields (which typically show a slow spatial frequency) in a narrow FOV is by definition challenging. Additionally, the long T1 at 7T, even with a relatively small flip angle $\left(8^{\circ}\right)$ in TFL, results in partial saturation of the CSF during the readout. Such a flip angle is necessary to achieve sufficient SNR, but it also implies reduced brainstem-4th ventricle contrast in the MT-TFL scans that further hinder registration. To tackle these challenges, we created an extended MT-TFL space by padding the scans with empty slices. The $\mathrm{T}_{1}$-weighted images were then resliced to the padded MT-TFL space, under the assumption that any movement between MP2RAGE and MT-TFL within-participant would be small and linear. These $\mathrm{T}_{1}$-weighted images, that covered a sufficient FOV to produce meaningful warps of the brainstem and have good GM/WM/CSF contrast (Marques et al., 2010), were then bias-field corrected (N4 algorithm) and a study-specific template of the $\mathrm{T}_{1}$-weighted scans to the MNI $0.5 \mathrm{~mm}$ space was created with an iterative diffeomorphic warp estimate using the buildtemplateparallel.sh script of the ANTS package. The MT-TFL scans were manually registered to the $\mathrm{T}_{1}$-weighted images with ITK-SNAP to correct for small displacements during the scan session. The transforms were concatenated. The MT-TFL scans were bias-field corrected (N4) to reduce intensity differences due to differing B1+ fields and projected over the single transform to the common space. A template was created from the average of their projection $(\mathrm{N}=20)$. The registration procedure is visualized in S-Fig. 1.

\subsubsection{Image processing}

To examine the feasibility of using a high-resolution MT-TFL approach to image the nucleus on an individual level in the face of potential physiological motion, individual NTS segmentations were carried out. A reference surrounding region was created by dilating the segmentations by $1 \mathrm{~mm}$ in all directions. The SNR $\left(S N R=\frac{\text { Mean }_{N T S}}{\text { Standard deviation }_{N T S}}\right)$ and $\mathrm{CNR}\left(\mathrm{CNR}=\frac{\text { Mean }_{N T S}-\text { Mean }_{\text {Surrounding region }}}{\text { Standard deviation }_{(\text {NTS }} \text { and Surrounding region) }}\right)$ were estimated to examine the localized signal characteristics. The CNR's standard deviation was estimated in the aggregate of both NTS and the surrounding region, to reduce the chance that human bias in the segmentation inflated the homogeneity of the ROI distribution. In practice, this did not seem to be a concern (Median Standard deviation $\mathrm{NTS}_{\mathrm{NTS}}=8.784$ and Median Standard deviation Surrounding region $=9.256$ ).

A NTS template was created in the MNI space. The MT-weighted atlas was inspected for a hyperintensity at the level of NTS; manual segmentation was then performed by NP. The localization was compared to a human brainstem histology-based atlas (Naidich et al., 2009). Considering that several studies have attributed fMRI activation to the NTS, we further decided to create a meta-analytical activation map and compare its localization to our template. First, studies were selected up to January 2019 in PubMed and Google Scholar with the keywords "solitary tract", "solitarius" AND "fMRI". The studies were examined to ensure that NTS-relating activation was reported; studies that did not report peak coordinates, thresholds and significant levels were excluded; this resulted in a total of 9 studies including $n=156$ participants (Supplementary Table 1). Conjunctive group differences across task conditions were excluded to ensure that duplicate data were not included. The peak coordinates and effect size of significant activations were extracted. The Seed-based $d$ Mapping software was used to perform a voxel-based meta-analysis. Briefly, the package translates the reported t-value to Hedge's effect sizes and filters them with an anisotropic kernel to 
A
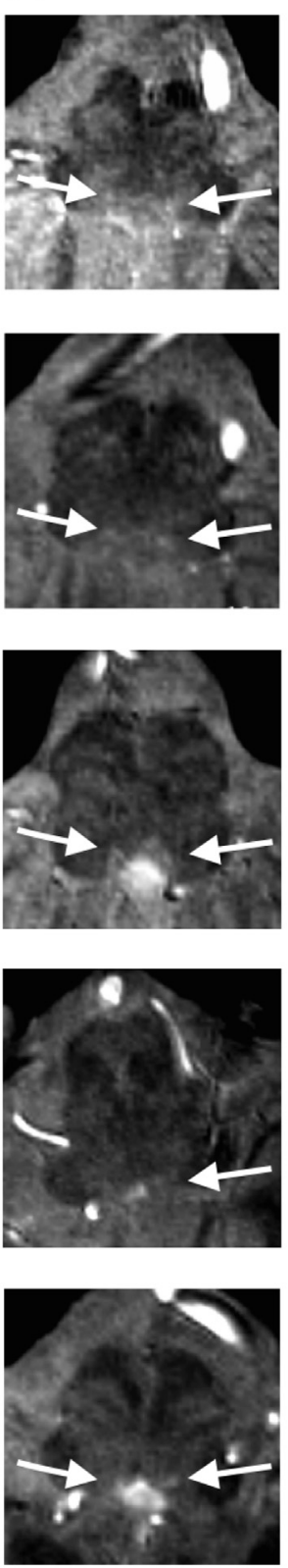

B
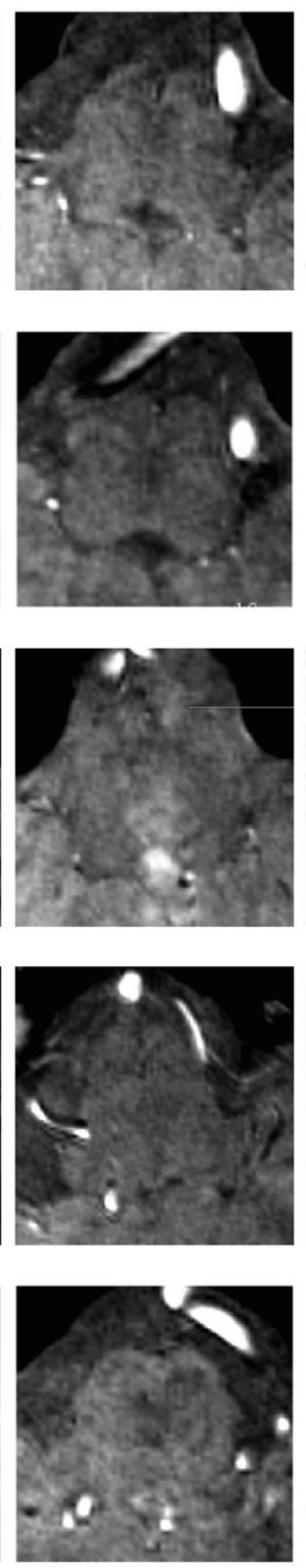

C
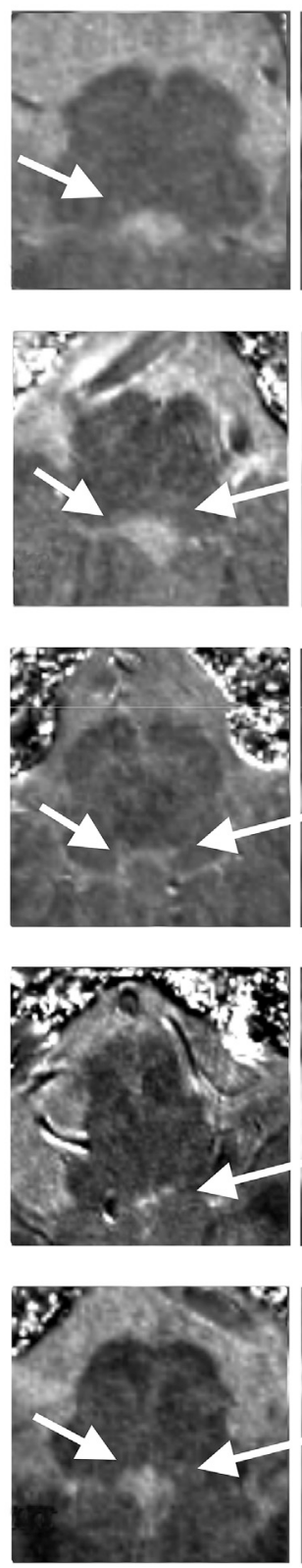

D
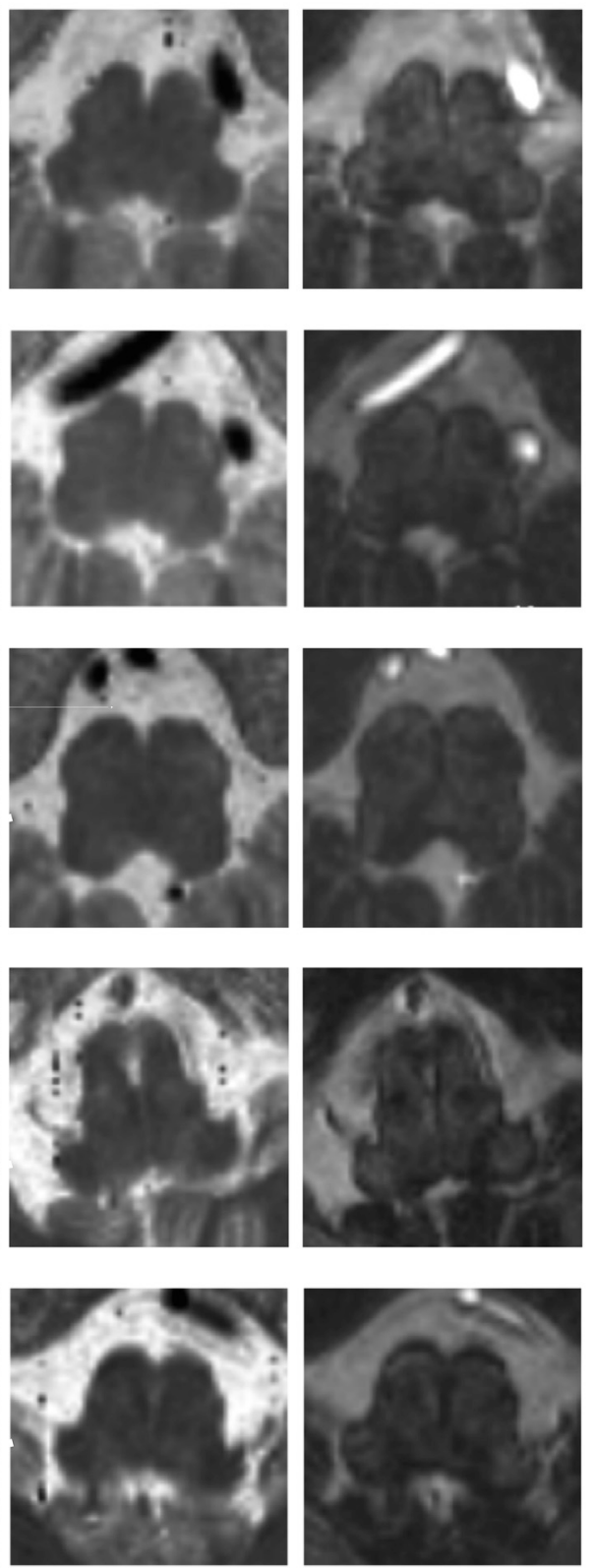

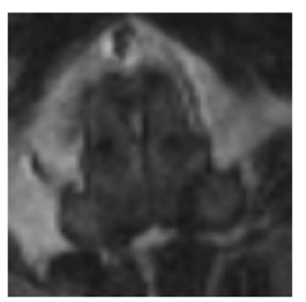

E
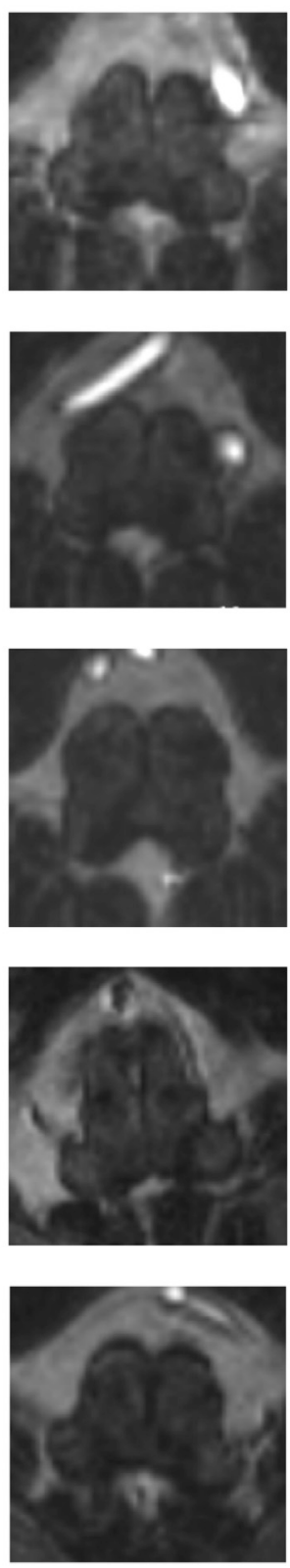

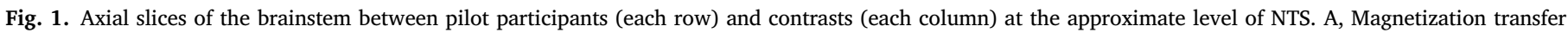

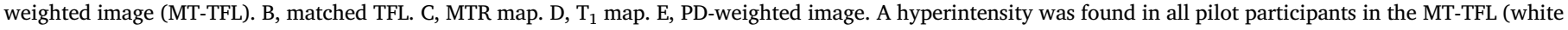
arrows). A similar hyperintensity was observed also in the MTR map, but not in the rest of the scans.

estimate the voxels relating to peak coordinates (Radua and Mataix-Cols, 2009; Rubia et al., 2014).

The NTS is a rod-like structure along the rostral-caudal axis. To examine the measurement variability across its axis, we performed individual NTS segmentations in the MNI space and found the coordinates of the individual peak voxel intensity per axial slice. The ratio of participants for whom a NTS-related hyperintensity was found was estimated per slice. To derive an estimate of the spatial spread of the distribution within each slice, we calculated the Mahalanobis distance $\left(D_{M}\right)$ compared to the median centroid, as

$D_{M}\left(x, x_{M}\right)=\sqrt{x-x_{M}^{T} S^{-1}\left(x-x_{M}\right)}$,

where $x$ are the coordinates of the individual peak voxel intensities per slice, $x_{M}$ is the median centroid of these coordinates per slice and $S$ is the covariance matrix.

\section{Results}

\subsection{Pilot results}

First the contrast specificity was examined in the pilot data of five participants. In all participants, a hyperintensity was detected in the MTTFL scan at the levels of NTS, albeit in one participant only on the left side (Fig. 1A). Furthermore, the contrast was not present in the matched reference TFL scan (Fig. 1B), but was observed in several scans at MTR map (Fig. 1C). This implies specificity to MT. No contrast was detected in the $\mathrm{T}_{1}$ map or the PD-weighted image (Fig. 1D-E).

We should note that in the MTR map, this hyperintensity was less readily observed. To quantify this, the hyperintensity was manually drawn and we calculated its CNR versus the surrounding region ( $1 \mathrm{~mm}$ in all directions) for MT-TFL and MTR. The MT-TFL showed a CNR of mean $=1.15(\mathrm{sd}=0.564)$, while the MTR map a CNR of mean $=0.667$ 
$(\mathrm{sd}=0.093)$.

The NTS-related hyperintensity in the MT-TFL was further compared to MT-TFL slices of LC and SNpc; all nuclei showed a similar slice intensity profile (Fig. 2).

\subsection{Localization}

Having established that a hyperintensity at the level of NTS exists, we examined its localization at the group-level. First, the NTS slices from the human brainstem atlas (Naidich et al., 2009) were extracted (Fig. 3A-C). Subsequently, we created a template from the whole dataset $(\mathrm{N}=20)$ and examined its localization compared to both the brainstem atlas and a meta-analytic NTS fMRI activation map. In total, we found 9 studies that fitted our criteria of reporting NTS activity. The meta-analytic map showed activation in the range of $\mathrm{z}=[-54,-43] \mathrm{mm}$ (Fig. 3D), in accordance with the expected position of NTS (Fig. 3B). A left and right cluster were observed, albeit with peaks at different levels (right: $\mathrm{z}=-52$, left: $\mathrm{z}=-44$ ). We subsequently created the template; a bilateral hyperintensity was observed across several slices between -47 and $-53 \mathrm{~mm}$ of the MNI space. The localization compared well to the atlas and the activation map (Fig. 3C-E). For visualization purposes, a NTS segmentation was drawn from the group-level template and imaged within a brainstem mesh (Fig. 3F).

\subsection{Individual variability}

We examined the individual NTS variability. Out of a total of 15 scans of the additional dataset, we were not able to detect a hyperintensity relating to NTS in 2 cases. The SNR within the NTS (signal SNR within each individual NTS segmentation in the native NTS space) was Mean $(\mathrm{sd})=17.40$ (2.57) a.u. compared to an SNR in the surrounding region of Mean (sd) $=13.42$ (1.64) a.u. The CNR compared to the surrounding region was also calculated as Mean (sd) $=1.10$ (0.13) a.u.

Following that, the frequency of the individual NTS signal for each slice was calculated (Fig. 4B). 50\% of the individual scans showed NTS contrast between -55 and $-44 \mathrm{~mm}$ in MNI space, with peak frequency at $-47 \mathrm{~mm}$. The distribution of the peak individual voxel intensity per axial slice was extracted (Fig. 4A) and the $\mathrm{D}_{\mathrm{M}}$ was calculated, as a measure of within-slice spatial spread (Fig. 4C). The $\mathrm{D}_{\mathrm{M}}$ was largely consistent between slices except for an increase in variability from $-42 \mathrm{~mm}$ rostrally.

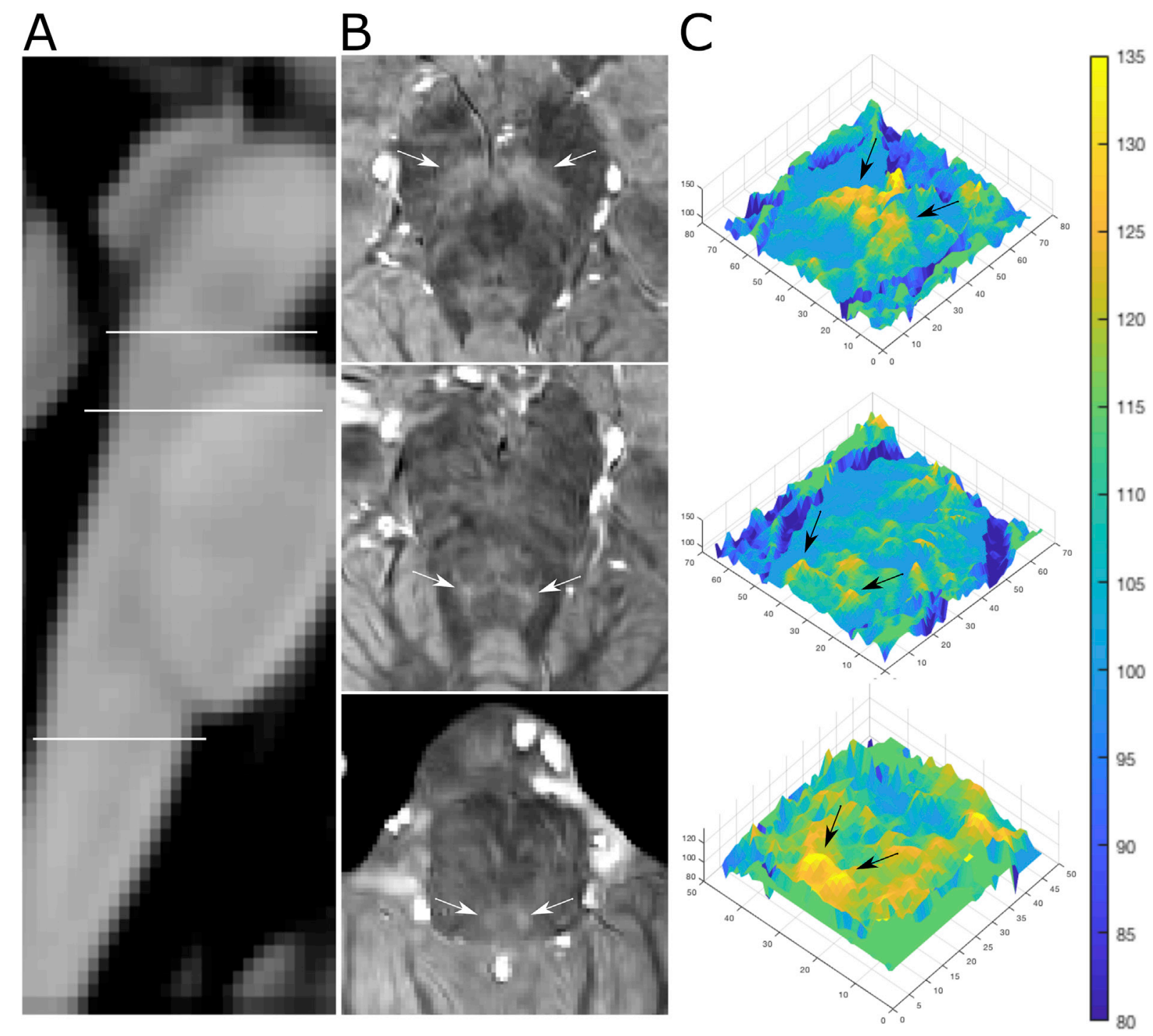

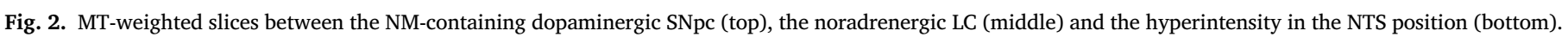

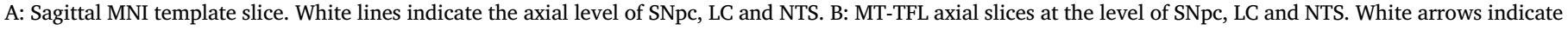
their position in the axial plane. C: Intensity profiles of each axial slice. Black arrows indicate the position of SNpc, LC and NTS. 
A

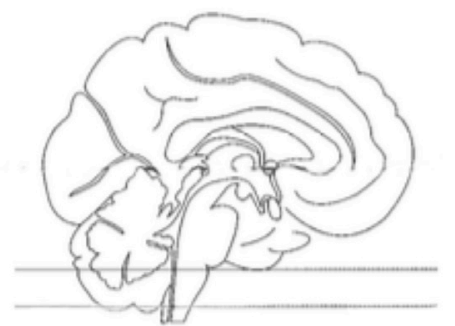

B

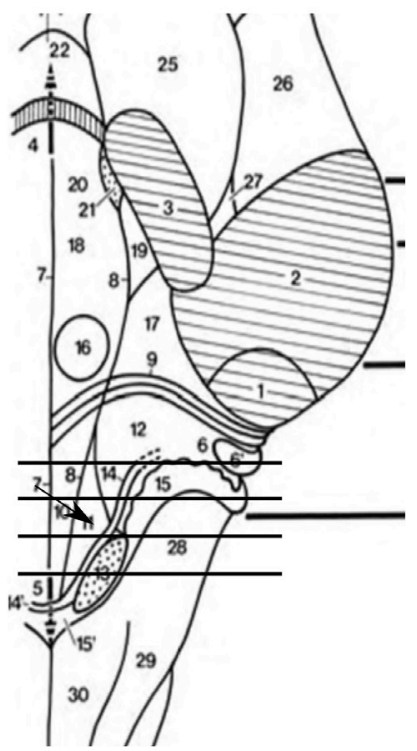

F

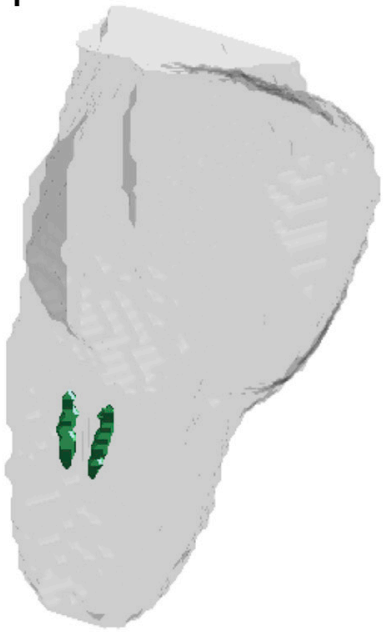

C
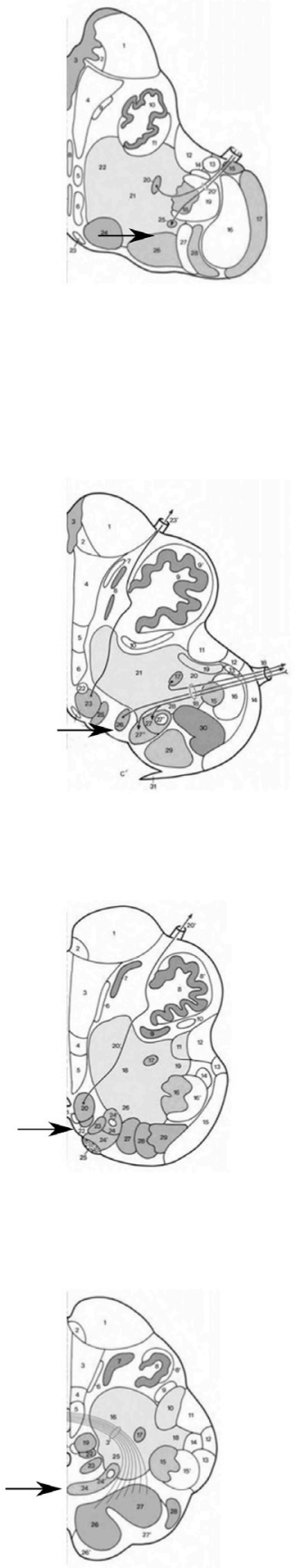

D

E
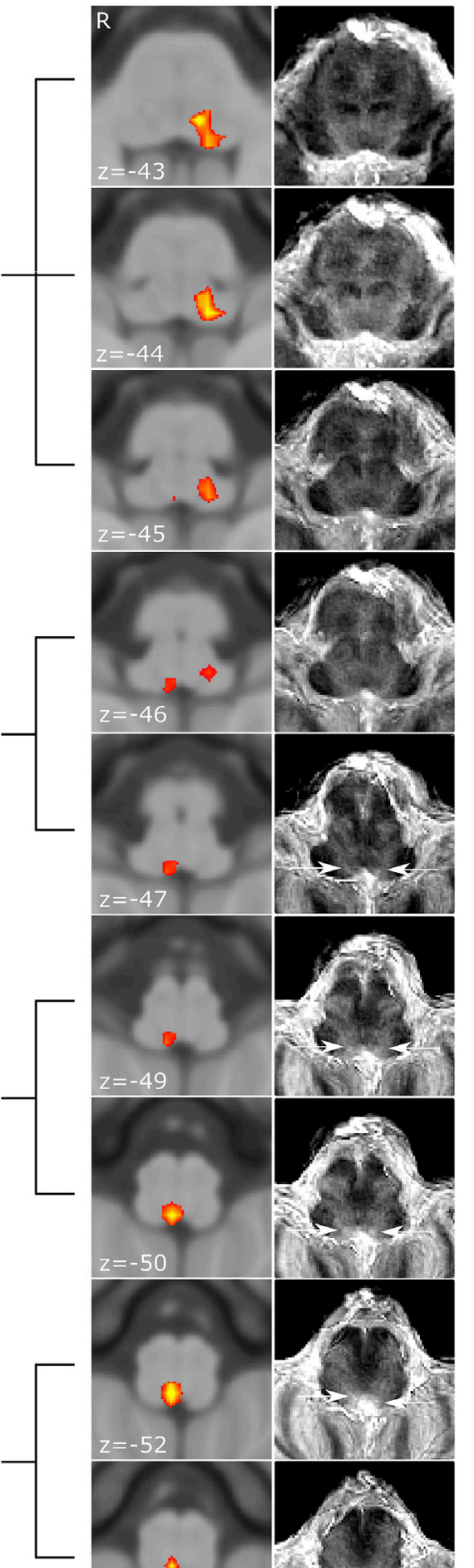
A

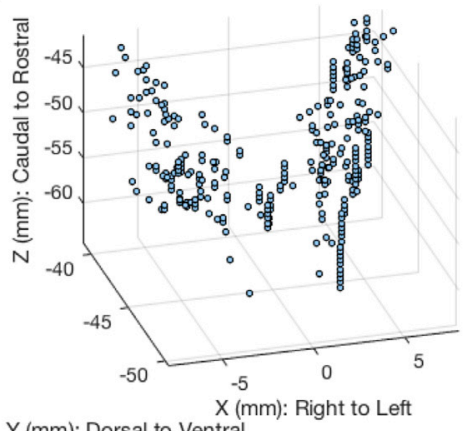

B

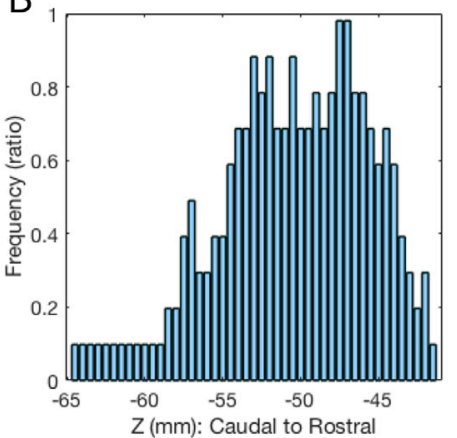

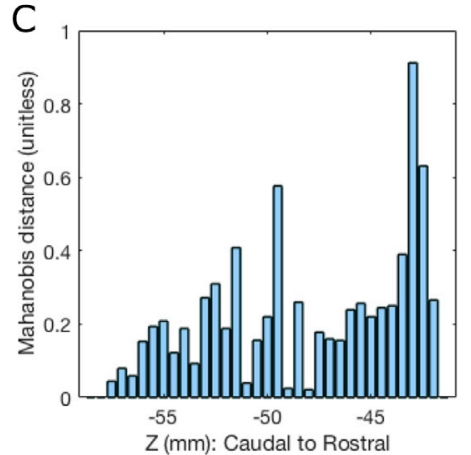

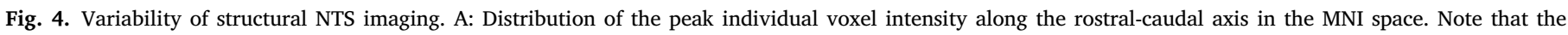

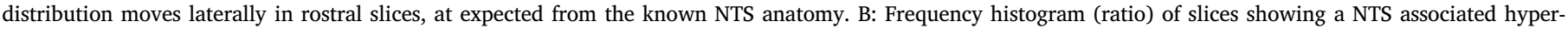

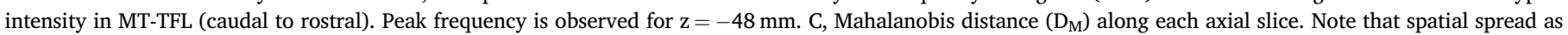
measured with $D_{M}$ is consistent between slices, except for the caudal part of the NTS (from $z=-59 \mathrm{~mm}$ on).

\section{Discussion}

In this study, we applied an efficient, high resolution MT-weighted sequence to structurally image the NTS, for the first time in vivo. We were able to demonstrate a well-localized contrast resulting in consistent localization across subjects. Furthermore, our template of the NTS showed good co-localization with a histological atlas and a meta-analytic fMRI-derived activation map. This approach may hold great promise for the neuroscientific as well as the clinical research field. The NTS is an important relay station between the vagus nerve and the brain and as such is involved in processing sensory information, visceral information, gastrointestinal but also cognitive information (Beckstead et al., 1980; Jones et al., 2012; Mello-Carpes and Izquierdo, 2013; Schwaber et al., 1980).

The NTS shows a high concentration of noradrenergic neurons (A1/2 subnuclei). Noradrenergic and dopaminergic nuclei, like the LC and SNpc, typically show reduced MT sensitivity compared to adjacent tissue (Bolding et al., 2013; Nakane et al., 2008; Priovoulos et al., 2018); we therefore hypothesized that a similar contrast would be found in the NTS. Indeed, we detected a hyperintense signal, similar to the one observed in LC and SNpc, specific to the MT-weighed image, but not in the matched TFL or $\mathrm{T}_{1}$ maps and PD images, supporting our suggestion that it relates to the noradrenergic NTS. While the LC/SNpc contrast itself has been suggested to positively correlate to NM concentration (Bolding et al., 2013; Keren et al., 2015), the putative mechanism that relates the hydrophobic NM to the exchange between semisolids and free water is not clear (Trujillo et al., 2016) and future studies should investigate the precise source of contrast.

The NTS showed good axial localization compared to both the atlas and the meta-analytic map of NTS-related fMRI activation. On the axial direction, we showed low imaging variability in terms of frequency and spatial spread in a set of axial slices ranging from $-58 \mathrm{~mm}$ to $-44 \mathrm{~mm}$ in MNI space which is consistent with the atlas and meta-analytic map description of NTS. However, at group level, the NTS template showed no hyperintensity rostrally to $\mathrm{z}=-47$ (Fig. $3 \mathrm{E}$ ). This is reflected in an increase in Mahalanobis distance rostrally to $\mathrm{z}=-44$ (Fig. 4C). Closer to the pons, the NTS is getting increasingly smaller, so this variability is to be expected (Naidich et al., 2009). We should note, however, that the meta-analytic map itself showed substantial variability on the ventral-caudal axis. This is not surprising, given the low temporal SNR of brainstem fMRI and the high-resolution needed for NTS. These results again highlight the need for a method that allows structural identification of the NTS.

A template, available in https://github.com/npriov/NTS was generated from the study population in the MNI space (Fig. 3F). While this was a study with relatively few participants $(\mathrm{N}=20$ in total), given that no other template exists as far as the authors are aware and that UHF scanners are still unavailable to a big part of the research community, this is an important first step towards facilitating research on the NTS.

On an individual basis, contrast in at least one slice was observed in 17 out of 20 cases (failure rate $=15 \%$ ), suggesting that the method consistently shows contrast in the NTS. In a previous study, we had used the same MT-TFL setup to image the LC at 7T (Priovoulos et al., 2018). Compared to those results, the NTS showed higher SNR $\left(\mathrm{Mean}_{\mathrm{NTS}}\right.$ $\left(\operatorname{sd}_{\text {NTS }}\right)=17.40$ (2.57) a.u. compared to $\operatorname{Mean}_{\mathrm{LC}}\left(\mathrm{sd}_{\mathrm{LC}}\right)=8.42(0.71)$ a.u $)$ but lower CNR though at reduced overall standard variability $\left(\right.$ Mean $_{\text {NTS }}$ $\left(\operatorname{sd}_{\mathrm{NTS}}\right)=1.10(0.13)$ a.u. compared to $\operatorname{Mean}_{\mathrm{LC}}\left(\mathrm{sd}_{\mathrm{LC}}\right)=1.52(0.34)$ a.u $)$. We should note that the study to image the LC had a low participant number $(\mathrm{N}=6)$, so any conclusions should be extracted carefully.

The above was achieved with a high-resolution image of almost $0.4 \mathrm{~mm}^{3}$ isotropic voxels in a relatively short TA of 4:30 min. Shortening TA is important both to reduce motion and increase the clinical and scientific relevance. Increasing the resolution further can be counterproductive, since motion becomes comparable to voxel size and TA becomes prohibitively long. The brainstem in particular is known to show a motion pattern related to the heart and respiration cycle, with a peak displacement of $\sim 0.185 \mathrm{~mm}$ (though potentially not uniform across the whole brainstem) (Soellinger et al., 2009). Online motion correction approaches, in the form of navigators to track the anterior-posterior brainstem displacement (Moghari et al., 2012) or motion cameras to track (respiration-related and not) motion (Gholipour et al., 2011) might be of benefit to push the resolution and decrease the failure rate further.

MT imaging at 7T imposes strict constraints: the SAR increases roughly quadratically, necessitating a decrease of the total saturation applied. To tackle this, a scheme of intensive saturation followed by a fast 3D readout was applied that allows high-resolution images under the saturation regime (Mougin et al., 2010) (more information on the choices made regarding the scheme can be found in our previous work (Priovoulos et al., 2018)). Even so, it is likely that additional contrast can be induced at $3 \mathrm{~T}$ by applying higher amplitude pulses. In our previous work, increased CNR was observed at 3T in the LC (Priovoulos et al., 2018). Additionally, the $\mathrm{B}_{1}+$ field, that scales linearly with MT, tends to be more inhomogeneous at 7T compared to 3T, confounding quantification of the MT effect across the brain. In this study, we focused on a small region and assumed a similar $\mathrm{B}_{1}+$ value between ROIs, given that $\mathrm{B}_{1}+$ tends to show slow spatial frequency changes. Researchers that are interested in extracting intensity values to examine clinical conditions, such as frequently done for example for the LC (Castellanos et al., 2015; Clewett et al., 2016), should correct for $\mathrm{B}_{1}+$ values (potentially as simple as a linear correction (Ropele et al., 2005)). High-field MT imaging also has several advantages: $\mathrm{T}_{1}$ recovery is slower, allowing longer MT-weighted readouts (van Gelderen et al., 2016). Furthermore, the bigger chemical shift between the macromolecular and free water pool reduces confounding direct saturation effects (Mougin et al., 2013). Finally, the SNR 
increase can be put to use in increased spatial resolution, which is needed to image these small structures (Table 3, Priovoulos et al., 2018). In this study, we did not examine if a similar setup can be used to visualize the NTS at 3T, since the nucleus has not been imaged before: the uncertainty of its position necessitated high-resolution imaging to identify consistent hyperintensities across axial slices. Future research can examine if our results can be translated to lower fields potentially with increased contrast, due to reduced SAR limitations.

This study has several limitations. First, the high-resolution required to image such small nuclei and the subsequent increase of noise, as well as the focus on visualization, led us to concentrate on the MT-TFL scan on its own. In cases where quantification of the MT effect is of interest, typical approaches include acquiring MT scans at different amplitudes and frequency offsets under the assumption of a 2-pool model in a steady state (Henkelman et al., 1993) or deriving MTR maps. An MTR map on its own, while sensitive to setup specifics, can be easily derived by acquiring a matched scan without MT and, due to its simplicity, has proven useful, particularly in clinical studies where scanning time is limited (Tambasco et al., 2011; Vavasour et al., 1998). The MTR maps in our initial pilot experiment did not seem to further improve the contrast. Future studies with increased SNR may achieve better contrast. Second, this is a study focused on examining the possibility of imaging the NTS at the individual level as well as its reproducibility between individuals. For that purpose, we found it useful to derive a group level template, which we make publicly available. We should note that this based on $\mathrm{N}=20$ individuals. It may be advisable to replicate this with a higher number of participants and/or specific populations.

The NTS consists of a set of nuclei that are involved in several vital and higher order cognitive functions, including breathing, heart rate and memory, but in vivo research of the NTS is hampered by the lack of imaging approaches. In this study, we applied an MT-based approach to image the NTS on an individual basis at UHF and provided a template to further facilitate research on the nucleus.

\section{Acknowledgements}

BAP is funded by the Netherlands Organisation for Scientific Research (NWO 016.Vidi.178.052) and the National Institutes of Health (R01MH111444, PI Feinberg).

\section{Appendix A. Supplementary data}

Supplementary data to this article can be found online at https://doi. org/10.1016/j.neuroimage.2019.116071.

\section{References}

Beckstead, R.M., Morse, J.R., Norgren, R., 1980. The nucleus of the solitary tract in the monkey: projections to the thalamus and brain stem nuclei. J. Comp. Neurol. 190, 259-282.

Bolding, M.S., Reid, M.A., Avsar, K.B., Roberts, R.C., Gamlin, P.D., Gawne, T.J., White, D.M., den Hollander, J.A., Lahti, A.C., 2013. Magnetic transfer contrast accurately localizes substantia nigra confirmed by histology. Biol. Psychiatry 73, 289-294.

Castellanos, G., Fernandez-Seara, M.A., Lorenzo-Betancor, O., Ortega-Cubero, S., Puigvert, M., Uranga, J., Vidorreta, M., Irigoyen, J., Lorenzo, E., Munoz-Barrutia, A., Ortiz-de-Solorzano, C., Pastor, P., Pastor, M.A., 2015. Automated neuromelanin imaging as a diagnostic biomarker for Parkinson's disease. Mov. Disord. 30, 945-952.

Clewett, D.V., Lee, T.-H., Greening, S., Ponzio, A., Margalit, E., Mather, M., 2016. Neuromelanin marks the spot: identifying a locus coeruleus biomarker of cognitive reserve in healthy aging. Neurobiol. Aging 37, 117-126.

Frangos, E., Ellrich, J., Komisaruk, B.R., 2015. Non-invasive access to the vagus nerve central projections via electrical stimulation of the external ear: fMRI evidence in humans. Brain Stimul. 8, 624-636.

Frangos, E., Komisaruk, B.R., 2017. Access to vagal projections via cutaneous electrical stimulation of the neck: fMRI evidence in healthy humans. Brain Stimul. 10, 19-27.
Garcia, R.G., Lin, R.L., Lee, J., Kim, J., Barbieri, R., Sclocco, R., Wasan, A.D., Edwards, R.R., Rosen, B.R., Hadjikhani, N., Napadow, V., 2017. Modulation of brainstem activity and connectivity by respiratory-gated auricular vagal afferent nerve stimulation in migraine patients. Pain.

Gholipour, A., Polak, M., van der Kouwe, A., Nevo, E., Warfield, S.K., 2011. Motion-robust MRI through real-time motion tracking and retrospective super-resolution volume reconstruction. Conf. Proc. IEEE Eng. Med. Biol. Soc. 2011, 5722-5725.

Henkelman, R.M., Huang, X., Xiang, Q.S., Stanisz, G.J., Swanson, S.D., Bronskill, M.J., 1993. Quantitative interpretation of magnetization transfer. Magn. Reson. Med. 29, 759-766.

Jones, R.B., McKie, S., Astbury, N., Little, T.J., Tivey, S., Lassman, D.J., McLaughlin, J., Luckman, S., Williams, S.R., Dockray, G.J., Thompson, D.G., 2012. Functional neuroimaging demonstrates that ghrelin inhibits the central nervous system response to ingested lipid. Gut 61, 1543-1551.

Keren, N.I., Taheri, S., Vazey, E.M., Morgan, P.S., Granholm, A.C., Aston-Jones, G.S., Eckert, M.A., 2015. Histologic validation of locus coeruleus MRI contrast in postmortem tissue. Neuroimage 113, 235-245.

Langley, J., Huddleston, D.E., Liu, C.J., Hu, X., 2016. Reproducibility of locus coeruleus and substantia nigra imaging with neuromelanin sensitive MRI. Magma 30, 121-125.

Little, T.J., McKie, S., Jones, R.B., D’Amato, M., Smith, C., Kiss, O., Thompson, D.G., McLaughlin, J.T., 2014. Mapping glucose-mediated gut-to-brain signalling pathways in humans. Neuroimage 96, 1-11.

Marques, J.P., Kober, T., Krueger, G., van der Zwaag, W., Van de Moortele, P.F., Gruetter, R., 2010. MP2RAGE, a self bias-field corrected sequence for improved segmentation and T1-mapping at high field. Neuroimage 49, 1271-1281.

Mello-Carpes, P.B., Izquierdo, I., 2013. The Nucleus of the Solitary Tract-> Nucleus Paragigantocellularis-> Locus Coeruleus- $>$ CA1 region of dorsal hippocampus pathway is important for consolidation of object recognition memory. Neurobiol. Learn. Mem. 100, 56-63.

Moghari, M.H., Hu, P., Kissinger, K.V., Goddu, B., Goepfert, L., Ngo, L., Manning, W.J. Nezafat, R., 2012. Subject-specific estimation of respiratory navigator tracking factor for free-breathing cardiovascular MR. Magn. Reson. Med. 67, 1665-1672.

Mougin, O., Clemence, M., Peters, A., Pitiot, A., Gowland, P., 2013. High-resolution imaging of magnetisation transfer and nuclear Overhauser effect in the human visual cortex at 7 T. NMR Biomed. 26, 1508-1517.

Mougin, O.E., Coxon, R.C., Pitiot, A., Gowland, P.A., 2010. Magnetization transfer phenomenon in the human brain at 7 T. Neuroimage 49, 272-281.

Naidich, T.P., Duvernoy, H.M., Delman, B.N., Sorensen, A.G., Kollias, S.S., Haacke, E.M., 2009. Duvernoy's atlas of the human brain stem and cerebellum: high-field MRI, surface anatomy, internal structure, vascularization and 3 D sectional anatomy. Springer Science \& Business Media.

Nakane, T., Nihashi, T., Kawai, H., Naganawa, S., 2008. Visualization of neuromelanin in the Substantia nigra and locus ceruleus at 1.5T using a 3D-gradient echo sequence with magnetization transfer contrast. Magn. Reson. Med. Sci. 7, 205-210.

Priovoulos, N., Jacobs, H.I.L., Ivanov, D., Uludag, K., Verhey, F.R.J., Poser, B.A., 2018. High-resolution in vivo imaging of human locus coeruleus by magnetization transfer MRI at 3T and 7T. Neuroimage 168, 427-436.

Radua, J., Mataix-Cols, D., 2009. Voxel-wise meta-analysis of grey matter changes in obsessive-compulsive disorder. Br. J. Psychiatry 195, 393-402.

Ramani, A., Dalton, C., Miller, D.H., Tofts, P.S., Barker, G.J., 2002. Precise estimate of fundamental in-vivo MT parameters in human brain in clinically feasible times. Magn. Reson. Imaging 20, 721-731.

Ropele, S., Filippi, M., Valsasina, P., Korteweg, T., Barkhof, F., Tofts, P.S., Samson, R., Miller, D.H., Fazekas, F., 2005. Assessment and correction of B1-induced errors in magnetization transfer ratio measurements. Magn. Reson. Med. 53, 134-140.

Rubia, K., Alegria, A.A., Cubillo, A.I., Smith, A.B., Brammer, M.J., Radua, J., 2014. Effects of stimulants on brain function in attention-deficit/hyperactivity disorder: a systematic review and meta-analysis. Biol. Psychiatry 76, 616-628.

Sasaki, M., Shibata, E., Tohyama, K., Takahashi, J., Otsuka, K., Tsuchiya, K., Takahashi, S., Ehara, S., Terayama, Y., Sakai, A., 2006. Neuromelanin magnetic resonance imaging of locus ceruleus and substantia nigra in Parkinson's disease. Neuroreport 17, 1215-1218.

Schwaber, J.S., Kapp, B.S., Higgins, G., 1980. The origin and extent of direct amygdala projections to the region of the dorsal motor nucleus of the vagus and the nucleus of the solitary tract. Neurosci. Lett. 20, 15-20.

Soellinger, M., Rutz, A.K., Kozerke, S., Boesiger, P., 2009. 3D cine displacement-encoded MRI of pulsatile brain motion. Magn. Reson. Med. 61, 153-162.

Tambasco, N., Belcastro, V., Sarchielli, P., Floridi, P., Pierguidi, L., Menichetti, C., Castrioto, A., Chiarini, P., Parnetti, L., Eusebi, P., Calabresi, P., Rossi, A., 2011. A magnetization transfer study of mild and advanced Parkinson's disease. Eur. J. Neurol. 18, 471-477.

Trujillo, P., Summers, P.E., Ferrari, E., Zucca, F.A., Sturini, M., Mainardi, L.T., Cerutti, S., Smith, A.K., Smith, S.A., Zecca, L., Costa, A., 2016. Contrast mechanisms associated with neuromelanin-MRI. Magn. Reson. Med. 78, 1790-1800.

van Gelderen, P., Jiang, X., Duyn, J.H., 2016. Effects of magnetization transfer on T1 contrast in human brain white matter. Neuroimage 128, 85-95.

Vavasour, I.M., Whittall, K.P., MacKay, A.L., Li, D.K., Vorobeychik, G., Paty, D.W., 1998. A comparison between magnetization transfer ratios and myelin water percentages in normals and multiple sclerosis patients. Magn. Reson. Med. 40, 763-768.

Yakunina, N., Kim, S.S., Nam, E.C., 2016. Optimization of transcutaneous vagus nerve stimulation using functional MRI. Neuromodulation 20, 290-300. 